\title{
Experimental study of the internal flow in freezing water droplets on a cold surface
}

\author{
Linn Karlsson $^{1} \mathbb{D} \cdot$ Henrik Lycksam ${ }^{1} \cdot$ Anna-Lena Ljung ${ }^{1} \cdot$ Per Gren $^{1} \cdot$ T. Staffan Lundström ${ }^{1}$
}

Received: 17 April 2019 / Revised: 9 October 2019 / Accepted: 10 October 2019 / Published online: 13 November 2019

(c) The Author(s) 2019

\begin{abstract}
The study of a freezing droplet is interesting in areas, where the understanding of build up of ice is important, for example, on wind turbines, airplane wings and roads. In this work, the main focus is to study the internal motion inside freezing water droplets using particle image velocimetry and to reveal if mechanisms such as natural convection and Marangoni convection have a noticeable influence on the flow within the droplet. The flow has successfully been visualized and measured for the first $25 \%$ of the total freezing time of the droplet when the velocity in the water is the highest and when the characteristic vortices can be seen. After this initial time period, the high amount of ice in the droplet scatters the PIV light sheet too much and the images retrieved are not suitable for analysis. Initially, it can be seen that the Marangoni effects have a large impact on the internal flow, but after about $15 \%$ of the total freezing time, the flow turns indicating increased effects of natural convection on the flow. Shortly after this time, almost no internal flow can be seen.
\end{abstract}

\section{Graphic abstract}

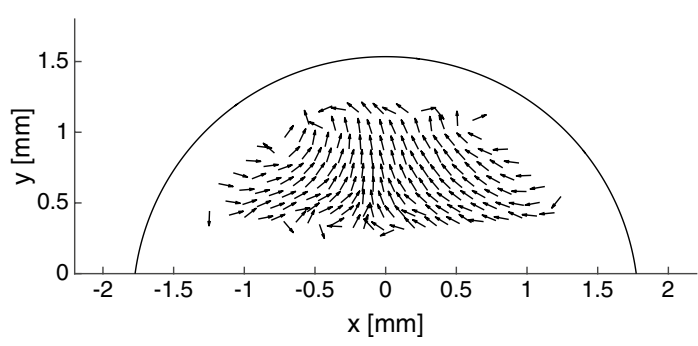

\section{Introduction}

Icing on wind turbines, airplane wings and roads is a major problem, since it often requires maintenance at high costs and may even cause damages and fatal accidents. There are many effective icing prevention methods to avoid these types of problems, but there are still a lot that is unknown about the ice itself. In this work, focus is on the flow within droplets that freeze on impact on the surface.

The overall freezing process of water droplets placed on cold surfaces has been visualized experimentally, using high-speed cameras (e.g., Jin et al. 2012, 2013b; Enríquez

Linn Karlsson

linn.karlsson@1tu.se

1 Div. of Fluid and Exp. Mechanics, Luleå University of Technology, 97187 Luleå, Sweden et al. 2012). Results presented include the volume expansion and freezing time during different ambient conditions. The volume expansion of the droplet results in a pointy tip and has been investigated both experimentally and numerically (e.g., Anderson et al. 1996; Enríquez et al. 2012; Snoeijer and Brunet 2012; Marín et al. 2014; Nauenberg 2014; Schetnikov et al. 2015). Anderson et al. (1996) studied a freezing droplet using a model that was able to reasonably capture the experimental solidified droplet with the cusp-like tip and inflexion point. Snoeijer and Brunet (2012), Marín et al. (2014) and Schetnikov et al. (2015) proposed numerical models to predict the angle of the conical tip and capture the volume expansion of the droplet. Zhang et al. (2017, 2018a) experimentally studied and statistically analyzed the icing nucleation characteristics of sessile water droplets. Zhang et al. (2018b) created a numerical model considering the 
supercooling effect and volume expansion effect and showed that they could reduce the deviation of freezing time from 30 to $10 \%$ for hydrophilic and hydrophobic surfaces.

By considering different cooling surfaces, interesting effects can be observed. For anti-icing purposes, a superhydrophobic surface can be used (e.g., Liu et al. 2007; Jin et al. 2013a; Oberli et al. 2014). For the interested reader, Oberli et al. (2014) presented a review on the freezing of a water droplet on these surfaces and their use in anti-icing applications. Other types of surfaces have also been considered (e.g., Huang et al. 2012; Jin et al. 2013a; Hao et al. 2014; Chaudhary and Li 2014). Huang et al. (2012) investigated the impact of contact angle (created using hydrophobic surfaces) on a freezing water droplet and concluded that a larger contact angle gives a longer freezing time. Hao et al. (2014) studied the freezing delay time and freezing time of a stationary droplet on surfaces with various roughness and wettability. They found that the surface roughness plays an important role for the nucleation. Jin et al. (2015) used an inclined cold surface to investigate the effects of droplet size and surface temperature on the impact, freezing, and melting process of a water droplet. Chaudhary and $\mathrm{Li}$ (2014) considered surfaces with different wettability that was subjected to rapid cooling to study the freezing process. They revealed that the freezing time is dependent on the droplet temperature at the pre-recalescence time and the surface wettability.

Kawanami et al. (1997) used a numerical model considering both surface tension and the density maximum at $4{ }^{\circ} \mathrm{C}$. The results were also validated with experiments. They found that both natural and Marangoni convection are important mechanisms for the internal flow. To the authors' knowledge Kawanami et al. (1997) is the only paper published considering the internal flow in a freezing water droplet experimentally. This suggests that there is a need to initiate additional experimental studies on the impact of the internal flow on a freezing water droplet.

Even though the internal flow during freezing of droplets seems to be a rather unexplored area, some studies have been performed on evaporation of droplets. Jin (2008) performed flow velocity measurements within water droplets using particle image velocimetry (PIV), at surfaces with temperatures ranging from 0 to $21.9^{\circ} \mathrm{C}$. The internal flow within the droplets was disclosed and velocities of the water estimated. Other authors also studied the internal flow numerically and experimentally (e.g., Ruiz and Black 2002; Hu and Larson 2006; Bhardwaj et al. 2009; Christy et al. 2010) with good results and additional work has been carried out on the effect from the surrounding flow (Ljung et al. 2015). More recently, Ma et al. (2019), investigated the flow field around the droplet using PIV and could from this calculate the aerodynamic force and adhesion force over the droplet. Hu and Larson (2006) and Xu and Luo (2007) aimed to investigate the influence from the Marangoni effect on the internal flow.
It was, however, revealed that the Marangoni number in their setups is 100 times lower than the theoretical one, which resulted in difficulties in capturing any influence on the flow from the Marangoni effect. This was corrected using clean interfaces, free of surfactants. Due to the success in the visualization of the internal flow in droplets on heated surfaces, this motivates the study of the internal flow inside freezing water droplets. The experimental method chosen in this work is PIV, which is a versatile nonintrusive, laser-based method, used in many applications to study fluid motion, e.g., PIV has been proven to be a reliable method when studying the internal flow in evaporating droplets (Jin 2008). The aim of this work is, therefore, twofolded: (1) to show that PIV can be used to visualize the movement of the water and estimate the velocities inside a freezing droplet and (2) to reveal if Marangoni effects and/or natural convection have a noticeable influence on the flow within the droplet.

\section{Method}

\subsection{Experimental setup}

Droplets were gently deposited on a $50.8 \mathrm{~mm}\left(2^{\prime \prime}\right)$ in diameter and $5 \mathrm{~mm}$ thick, cold sapphire plate (aluminium oxide, $\mathrm{Al}_{2} \mathrm{O}_{3}$ ) using a pipette. The motivation for using the sapphire plate as the cooling surface is the transparency in combination with a high thermal conductivity $(k=46 \mathrm{~W} / \mathrm{mK}$, Incropera et al. 2013) allowing the laser sheet to pass through the droplet from beneath. The high $k$ implies a faster cooling of the surface in comparison to other transparent materials as, for example, plate glass or pyrex glass with $k=1.4 \mathrm{~W} / \mathrm{mK}$ (Incropera et al. 2013). The pipette (ThermoFisher, Finnpipette $\mathrm{F} 11$ to $10 \mu \mathrm{L}$, micro) was set at releasing $10 \mu \mathrm{L}$ droplets and was kept in place by an optical rail to make the position of the pipette tip repeatable. The cooling surface was resting on an aluminium holder in direct contact with a Peltier cooler with the warm side submerged in a box with cold circulating water (closed system, connected to a tank with ice water, held at $T=3.9 \pm 1.3^{\circ} \mathrm{C}$ ). To guide the incoming light underneath the droplet, a prism was placed in a central hole of the aluminium holder and a channel in the holder allowed the light sheet to pass through to the droplet. A PMMA (plexiglass) chamber was positioned around the sapphire plate to protect the area around the cooling surface from disturbances from surrounding airflows in the room. Four holes were made on the chamber; one on the top for the pipette to enable the release of the droplet to the surface, one on the right side to allow the laser light go directly to the prism, one on the left side for the camera to get a clear view of the setup and one on the back to mount the hygrometer to the plexiglass chamber. Before each experiment, the sapphire plate was cleaned using propanol $\left(\mathrm{C}_{3} \mathrm{H}_{7} \mathrm{OH}\right)$ and 
deionized (DI) water, and then dried with lens paper. A schematic diagram of the experimental setup can be seen in Fig. 1.

The liquid used in the experiments was DI water kept at room-temperature, $T_{\text {water }}=21.1 \pm 1.4{ }^{\circ} \mathrm{C}$ and was seeded with fluorescent particles of Rhodamine B (microParticles GmbH PS-FluoRed, aqueous dispersion $25 \mathrm{mg} / \mathrm{mL}$ ) with a diameter of $3.16 \mu \mathrm{m}$. The Stokes number (Stk) was calculated to determine if the particles were suitable for these experiments. The relaxation time of the particles varies between 0.33 and $0.59 \mu$ s for the setup, a typical diameter is $3.42 \mathrm{~mm}$ (case 1 in Table 1) and the velocity in the 10 cases varies typically between 0 and $1 \mathrm{~mm} / \mathrm{s}$. This results in a Stk $<5 \times 10^{-7}$ or, for the most part much less. Since Stk $\ll 1$, the conclusion is that the particles follow the flow well. The amount of particles in the water was based on the guidelines $>6$ particles per interrogation area. The concentration of particles was eventually set to $0.98 \mathrm{ml}$ DI water and $0.02 \mathrm{ml}$ seeding particle suspension. 20 droplets were weighted on a highly sensitive scale and the droplet mass was found to be $9.98 \pm 0.13 \mathrm{mg}$.

The temperature of the air inside the plexiglass chamber and the temperature of the sapphire plate were monitored by two thermocouples of $\mathrm{T}$ and K-types, respectively. The laser was a continuous $50 \mathrm{~mW} 532 \mathrm{~nm} \mathrm{Nd:YAG} \mathrm{(Altechna}$ Co Ltd) connected to a half waveplate, a polarizing beam splitter (cube) and a beam dump. These components were used to adjust the amount of light transmitted to the droplet. A cylinder lens assembly from Dantec Dynamics created and focused the light sheet to a thickness of $<0.4 \mathrm{~mm}$ thick. A 12 $\mathrm{mm}$ thick window placed on a rotation table was used to fine tune the position of the sheet to the center of the droplet (up and down to be able to adjust the light sheet to move sideways in the droplet), see Fig. 1. The benefit of illuminating
Table 1 Values of the droplets geometry and freezing times

\begin{tabular}{lllcl}
\hline Case & $\begin{array}{l}\text { Droplet } \\
\text { height }(\mathrm{mm})\end{array}$ & $\begin{array}{l}\text { Droplet } \\
\text { radius }(\mathrm{mm})\end{array}$ & $\begin{array}{l}\text { Contact area at } \\
\text { surface }\left(\mathrm{mm}^{2}\right)\end{array}$ & $\begin{array}{l}\text { Freezing } \\
\text { time }(\mathrm{s})\end{array}$ \\
\hline 1 & 1.42 & 1.71 & 9.15 & 16 \\
2 & 1.50 & 2.01 & 12.7 & 13 \\
3 & 1.56 & 1.83 & 10.5 & 13 \\
4 & 1.59 & 2.00 & 12.6 & 20 \\
5 & 1.59 & 1.67 & 8.76 & 17 \\
6 & 1.65 & 1.83 & 10.5 & 20 \\
7 & 1.92 & 1.58 & 7.81 & 30 \\
8 & 1.92 & 1.53 & 7.39 & 34 \\
9 & 1.93 & 1.52 & 7.26 & 34 \\
10 & 2.16 & 1.09 & 3.75 & 49 \\
\hline
\end{tabular}

the droplet from beneath is that it guides the laser light in a better way then if, for example, the light was coming from above, which would scatter the light more. Another advantage with the chosen tactic is that it allows for a good view of the symmetry line in the droplet. A CMOS camera (IDS $\mu$ Eye) with a spatial resolution of $1280 \times 1024$ pixels and pixel size $5.3 \times 5.3 \mu \mathrm{m}^{2}$ together with a Navitar long distance microscope captured images of the particles. The measurements were performed at a frequency of $50 \mathrm{~Hz}$ and with an exposure time $5 \mathrm{~ms}$ during the full freezing process. The recording times were dependent on the freezing time for the droplet defined, as the time from when the droplet hits the surface until the pointy tip has appeared. The droplet was released from a distance of $3.9 \mathrm{~mm}$ above the surface. This height was carefully determined, since it was the shortest possible distance for the droplet to be completely released from the pipette before touching the surface. The velocity of the droplet when it hit the surface was about $77 \mathrm{~mm} / \mathrm{s}$.

Fig. 1 Schematic diagram of the experimental setup

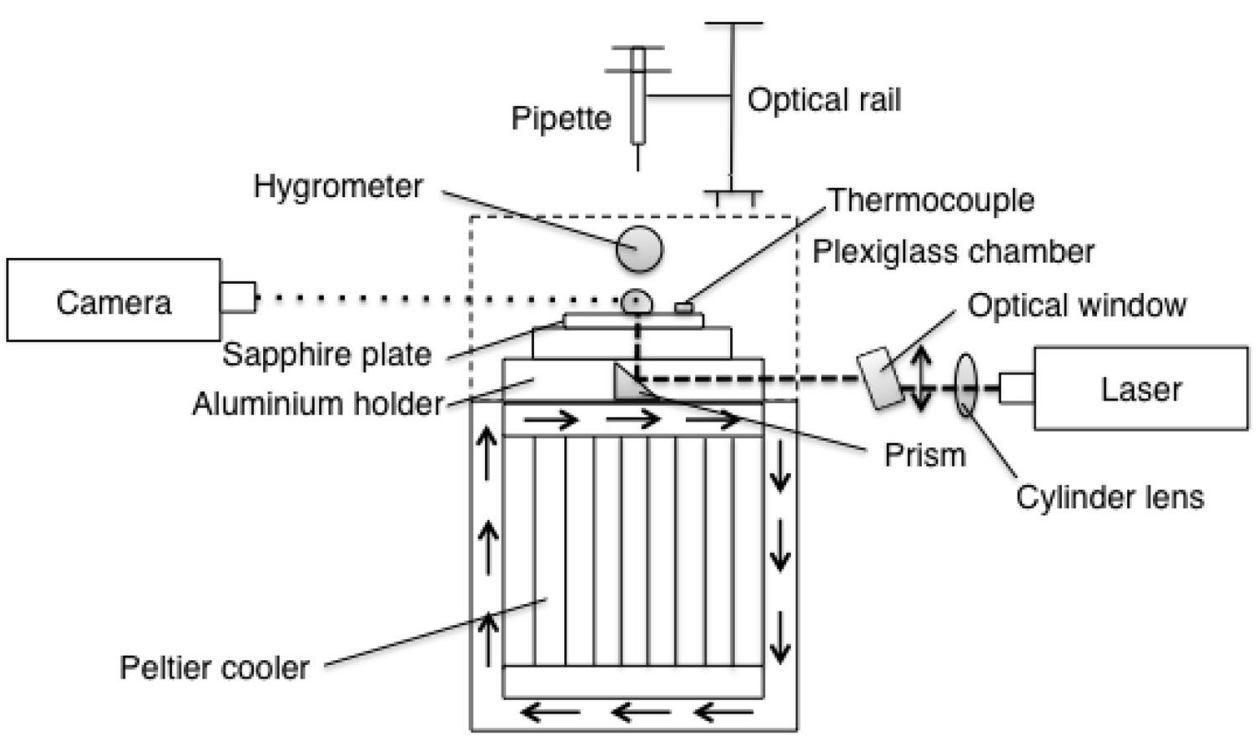




\subsection{Frost formation}

Initial experiments indicated that a roughness on the cooling surface was necessary to initialize the freezing process of the droplet without delay otherwise the droplets would become supercooled instead. Since the sapphire plate is very smooth, this roughness had to be added to start the nucleation within the droplet. Frost is relatively easy to create and is more or less always present when working with freezing temperatures in nature due to the relative humidity in the air; therefore, it was used to generate the required roughness on the surface.

The frost was created by letting pressurized air pass through a container filled with water and into the closed chamber surrounding the cooling surface. A regulator was used to adjust the flow rate of the incoming air and as the air passed the water container it was humidified. The temperature of the surface, as well as the air and the relative humidity inside the chamber was monitored to create a layer of frost as similar as possible during each freezing. These values were, $T_{\text {plate }}=-8.08 \pm 0.12{ }^{\circ} \mathrm{C}, T_{\text {chamber }}=16.7 \pm 1.7$ ${ }^{\circ} \mathrm{C}$ and $\mathrm{RH}=50.4 \pm 4.5 \%$, respectively.

\subsection{Experimental procedure}

The following statements can briefly summarize the experimental procedure:

1. The cooling of the surface starts.

2. At $T_{\text {plate }}=-8.0^{\circ} \mathrm{C}$ (visually determined from the computer screen), the pressurized air was switched on and turned off again when $\mathrm{RH}$ was around $50 \%$. This took about $60 \mathrm{~s}$.

3. The pipette was filled with the DI water and seeding particle suspension.

4. The camera and the laser were switched on. The position of the light sheet was fined tuned to the centre of the droplet (while the droplet was still hanging from the pipette).

5. The droplet was released when $T_{\text {plate }}$ reached $-8.0{ }^{\circ} \mathrm{C}$ again (visually determined from the computer screen), which occurred approximately $30 \mathrm{~s}$ from when the pressurized air was switched off.

6. The cooling was turned off when the droplet was completely frozen.

7. The surface was cleaned and dried when $T_{\text {plate }}>0.0^{\circ} \mathrm{C}$ and at $T_{\text {plate }}=4.0^{\circ} \mathrm{C}$ a new experiment could begin.

\subsection{Data processing}

The images were processed in the GUI-based open-source tool, PIVlab, for DPIV analyses in MATLAB (Thielicke and Stamhuis 2014). Four image pre-processing techniques were used to improve the measurement quality and to facilitate the visual inspection: contrast-limited adaptive histogram equalization - to enhance contrast in the image, high-pass filtering - to sharpen the image and remove background signal, intensity capping - which reduces the increased influence of very bright particles and Adaptive Wiener-for noise reduction (Thielicke and Stamhuis 2014). A multi-pass correlation scheme with decreasing window size and window off-set was used to calculate the particle displacements, the interrogation window size was $64 \times 64$ pixels (first pass) decreasing to $32 \times 32$ pixels (second pass) and finally $16 \times$ 16 pixels (third pass) with adaptive window shift, all with an overlap of 50\%. The standard FFT algorithm was used for the cross correlation, with a three-point Gaussian peak fit to estimate the sub-pixel displacement. A standard deviation filter and a local median filter were also applied at this point.

A known issue when working with PIV measurements on droplets on surfaces is that the refraction of light at the droplet surface creates a problem when measuring the flow field inside the droplet. This can be corrected by a mapping function between the points on the image plane and object plane using a method derived by Kang et al. (2004) and Minor et al. (2007). This is based on the ray tracing method and can be divided into two sub-methods, the image mapping method and the velocity mapping method. The first uses a technique to restore distorted images, and the second maps the velocity vectors from the original particle images directly onto the object plane without image restoration. Since the velocity mapping method is recommended by Kang et al. (2004) it is applied in this work. The first step in the method is to find the edge or boundary of the droplet using a least-square curve fitting. This marks the area for where the velocity mapping method is used. The images of the freezing droplets are then evaluated using the PIV-software and exported into MATLAB. Using the data from the edge detection and the data from the PIV-software, the velocity mapping method then directly map the velocity vectors obtained in the image plane to the object plane and the output is a corrected image of the velocity field in the droplet. Note that when using this method the centre region is well restored and provides accurate flow images of this region, but it does not work well in the outer region when $\eta_{\mathrm{o}} / R>0.75$, where $\eta_{\mathrm{o}}$ is the radial distance from the centre to a point in the bottom plane of a hemispherical lens and $R$ is the radius of the hemispherical lens. This means that there will be an area close to the edge that will be difficult to restore and retrieve a good image of because of total internal reflection (Kang et al. 2004). By preliminary studies it could be seen that most of the action takes place in the beginning of the freezing process and that most of the movement has stopped almost completely within in a few seconds. Also, after this period of time the ice scatters the light too much and the images retrieved are not suitable for 
analysis. Therefore, the results presented here consider the first $25 \%$ of the full freezing time.

\subsection{The droplets}

The height, radius, contact area to the cooling surface and freezing time of 10 droplets that was deposited in the setup presented above, varies according to Table 1 . The mean and standard deviation of these are $1.7 \pm 0.2 \mathrm{~mm}, 1.7 \pm 0.3 \mathrm{~mm}$, $9.0 \pm 2.7 \mathrm{~mm}^{2}$ and $24.6 \mathrm{~s} \pm 11.8 \mathrm{~s}$, respectively. The height of the droplet is defined as the height between the cooling surface and the apex of the droplet along the symmetry line, the droplet radius is defined as the radius at the cooling surface and the contact area to the cooling surface is calculated using the radius of the droplet.

\subsection{Uncertainty analysis}

Uncertainties during the measurements can be divided into two categories-systematic, or bias, errors that arise usually from to the measuring instruments and random errors due to unknown or unpredictable changes in the experiments (Coleman and Steele 2009).

The main sources to systematic errors originates, for example, from the pipette technique, i.e., including errors in the mixing of water with the seeding particles, the reading of the instruments during freezing and the positioning of the camera. These errors are difficult to detect, since they always force the result in the same direction and may, therefore, impact the outcome of the experiments significantly, but careful planning and execution of experiments can minimize these errors. The correlation error is normally 0.1 pixel (Westerweel 2000).

The random errors stem mainly from the release of the droplet or from the frost layer created on the surface that results in droplets with different contact angles and because of this different freezing times. To get an estimate of the random errors a repeatability test can be performed, see Coleman and Steele (2009). Ten experiments are considered, where the magnitude of the corrected velocities in the $y$-direction along the symmetry line between bottom and apex of the droplet is studied. Since the droplets vary in height the interesting points is what happens just above the freezing front, i.e., the lowest value of the corrected data set and at the top of the corrected data in each case. Also, the points at 25,50 and $75 \%$ above the freezing front is determined, see the inserted figure in Fig. 2 for the locations exemplified for case 1 . The freezing front is defined as the area, where the water and ice meet in the droplet. To minimize the effect of freezing time the times chosen for the repeatability test are scaled with the total freezing time. The times studied are $t=0.09 t_{\text {total }}$ and $0.19 t_{\text {total }}$, i.e., 9 and $19 \%$ of the full freezing times, and these times are chosen because of the different direction of flow (up and down, respectively, along the symmetry line) to be able to study the error at two different conditions during the freezing process. In Table 2 the precision errors with a $95 \%$ confidence interval for the 5 points chosen are shown and it can be seen that the errors are below, or mostly well below $1.5 \%$, suggesting that the

Table 2 Precision error in five points along the symmetry line for $t=0.09 t_{\text {total }}$ and $0.19 t_{\text {total }}$

\begin{tabular}{lll}
\hline Position & $t=0.09 t_{\text {total }}(\%)$ & $t=0.19 t_{\text {total }}(\%)$ \\
\hline Above freezing front & 0.54 & 0.23 \\
$25 \%$ & 1.27 & 0.44 \\
$50 \%$ & 0.021 & 0.16 \\
$75 \%$ & 1.48 & 0.74 \\
Top & 0.076 & 0.62 \\
\hline
\end{tabular}

Fig. 2 Mean velocity in the five points used in the repeatability study at $9 \%$ and $19 \%$ of the full freezing times in the 10 cases studied. The precision error with a $95 \%$ confidence interval in each point is shown with error bars. In the inserted figure, the locations of the points used in the repeatability study are exemplified for case 1 . Point 1 is located just above the freezing front and point 5 is located at the top of the corrected data. Points 2, 3 and 4 are found inbetween point 1 and 5

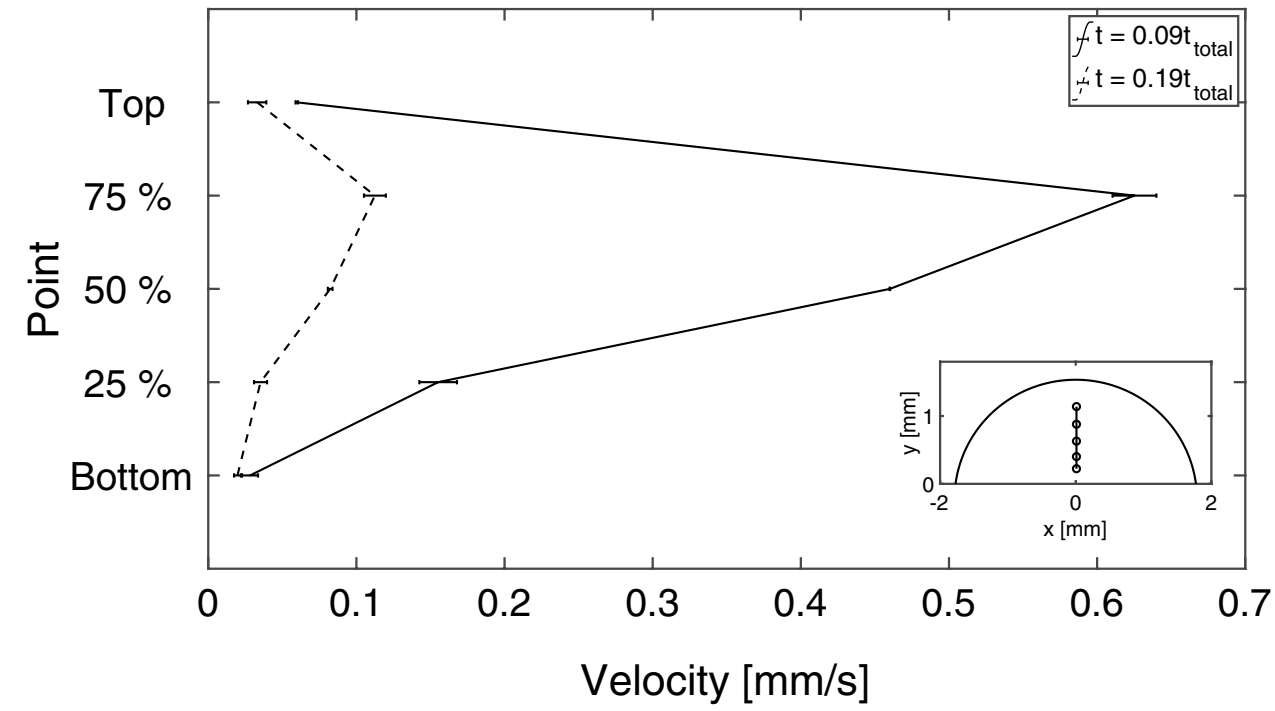


random errors are small regarding the velocities on the symmetry line. This can also be seen in Fig. 2, where the mean velocity in the five points is shown together with the precision error presented using error bars. This means that the velocities in the droplet are in fact comparable in each case despite their differences in appearance, and that one of the droplets can be selected for further study.

\section{Results and discussion}

In this part results regarding the freezing front and the flow field within one droplet is presented and the frost formation is discussed. As exemplified in Fig. 2, the overall flow field in the 10 droplets is comparable, and therefore, one droplet (case 1) can be chosen for further study.

\subsection{The freezing front}

The height of the freezing front, defined as the area, where the water and ice meet in the droplet, is studied in the performed experiments in three cases (case 1,5 and 10) and compared to experiments by Jin et al. (2013b) and simulations by Karlsson et al. (2018). Note that the setups are somewhat different and that the volumes of the droplets are not identical. The volumes of the droplets in the experiments performed by Jin et al. and the simulations by Karlsson et al. are $9.32 \mu \mathrm{l}$ as compared to $10 \mu \mathrm{l}$ in this work (for exact details about the droplet sizes in this work, see Table 1). Both the times and the heights are scaled with the total freezing time and the total height of the droplet for each case. In Fig. 3 it can be seen that the freezing front is approximately linear for the first $25 \%$ of the freezing process investigated (data up to 35 and $38 \%$ of the freezing processes is used from the works by Jin et al. 2013b; Karlsson et al. 2018, respectively) and that the similarities between the three cases are strikingly

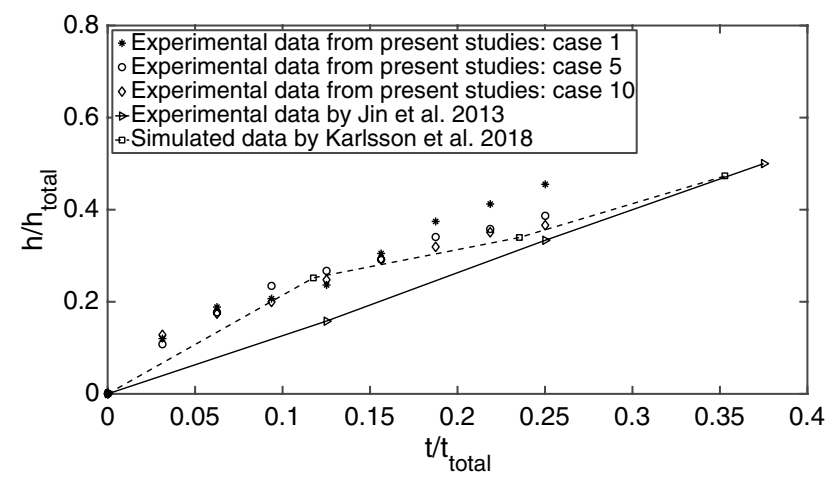

Fig. 3 Height of the freezing front at different times scaled with the corresponding droplet heights and freezing times in three works: from the present experiments (case 1,5 and 10), experimental data by Jin et al. (2013b) and from simulations by Karlsson et al. (2018) close despite the differences between them. This suggests that the freezing is caused by pure heat conduction from the cooling surface.

\subsection{Internal flow patterns in the freezing water droplet}

At $t=0 \mathrm{~s}$, the droplet hits the surface and during the first second the water in the droplet moves in a chaotic way, influenced by the release from the pipette and the collision with the surface. During this time period, no data is extracted from the images. At $t=1 \mathrm{~s}\left(t=0.06 t_{\text {total }}\right)$, it is clear that the freezing has already started and vortices emerge on both sides of the symmetry line of the droplet moving up along symmetry line and down along the droplet-air surface to the cooling surface, see Fig. 4a. When the droplet has frozen to about half its volume (at approximately $t=0.25 t_{\text {total }}$ ) these vortices start to decay in strength and the flow is slowed down. As the freezing continues, no vortices can be seen and there is almost no flow in the remaining water. In Fig. 5, the magnitude of the velocity in the $y$-direction along the symmetry line at $t=1$ to $4 \mathrm{~s}\left(t=0.06 t_{\text {total }}\right.$ to $\left.0.25 t_{\text {total }}\right)$ can be seen. The highest velocities can be found in the beginning of the freezing process, but at $t=2.5 \mathrm{~s}\left(t=0.16 t_{\text {total }}\right)$ the flow starts to shift, see Fig. 4d, where the flow becomes chaotic and this is also reflected in the velocity, since it approaches zero at this point. After this time, the flow has changed direction and an increase in velocity can be seen again at $t=3 \mathrm{~s}$ $\left(t=0.19 t_{\text {total }}\right)$ before it slows down again at $t=4 \mathrm{~s}$ and the velocities approaches zero. In Fig. 6 the magnitude of the velocity in the $y$-direction along four lines in the droplet are displayed, corresponding to the times in Fig. 4, and here it is possible to see how the velocity varies throughout the droplet. Note that the lines are at different locations at each time and that the velocity scales varies. When $t=1 \mathrm{~s}$, it can be seen that the highest velocities are found closer to the surface of the droplet, but just before the shift in direction of the flow the velocities are evened out in the droplet. After the shift (at $t=2.5 \mathrm{~s}$ ), the highest velocities can again be found closer to the surface, but shortly after the velocities are once more evened out in the droplet.

If following the theory regarding Marangoni convection, the vortices are driven by the differences in surface tension arising from the large temperature differences at the droplet surface. According to Kawanami et al. (1997) the Marangoni convection has larger effect on the flow than natural convection caused by density differences. The initial temperature difference in the freezing water droplets is about 30 ${ }^{\circ} \mathrm{C}$ between the water and the cooling surface. The surface tension for water in contact with air increase with decreasing temperature, which means that the water will be pulled to regions with higher surface tension (hence low temperature). For the case of a freezing droplet, this means that the water 


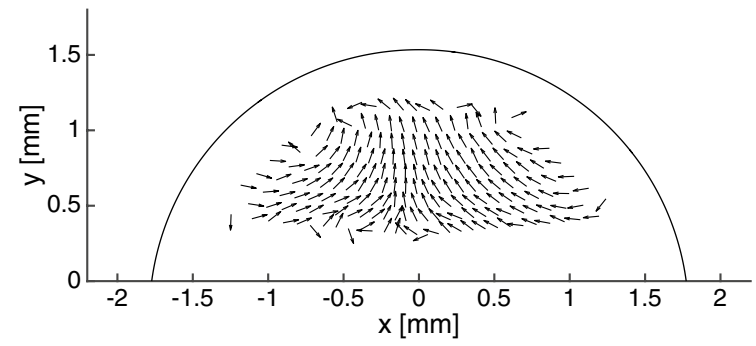

(a) $t=1 \mathrm{~s}\left(t=0.06 t_{\text {total }}\right)$

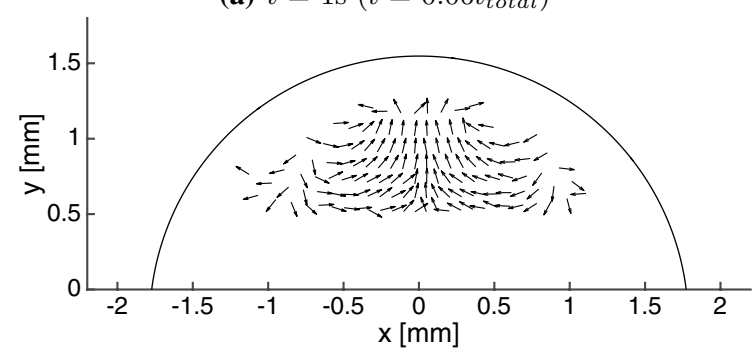

(c) $t=2 \mathrm{~s}\left(t=0.13 t_{\text {total }}\right)$

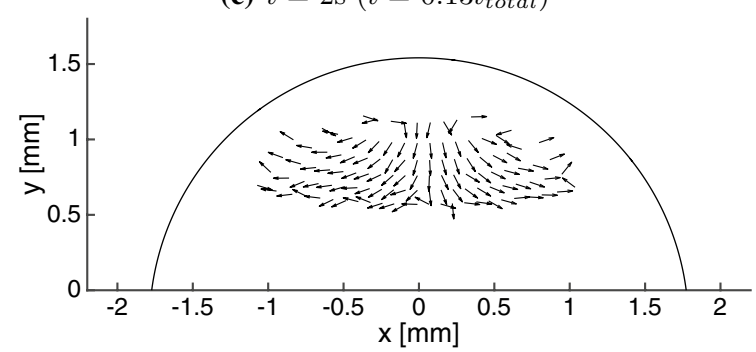

(e) $t=3 \mathrm{~s}\left(t=0.19 t_{\text {total }}\right)$

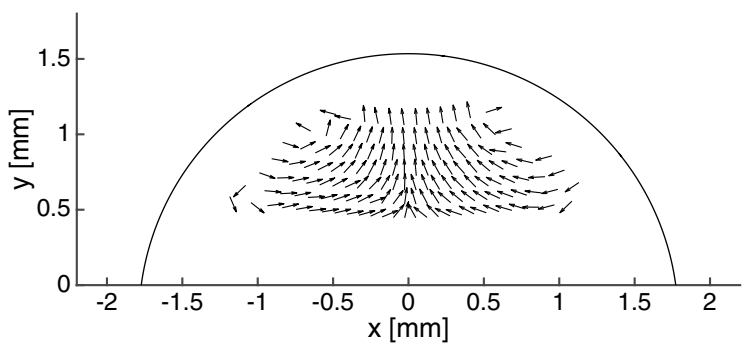

(b) $t=1.5 \mathrm{~s}\left(t=0.09 t_{\text {total }}\right)$

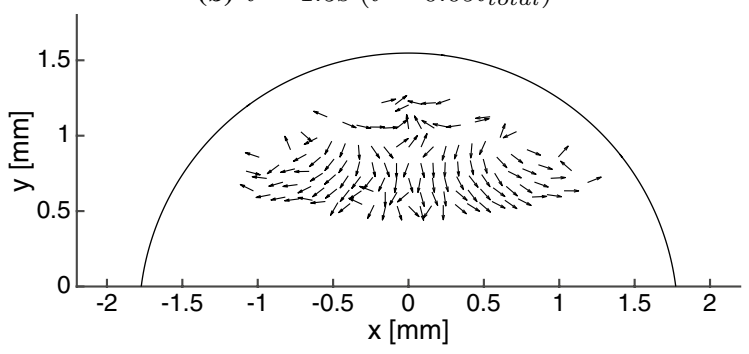

(d) $t=2.5 \mathrm{~s}\left(t=0.16 t_{\text {total }}\right)$

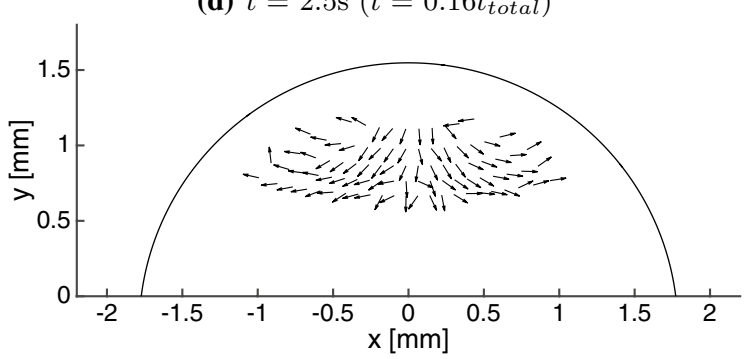

(f) $t=4 \mathrm{~s}\left(t=0.25 t_{\text {total }}\right)$

Fig. 4 Internal flow patterns with normalized velocity vectors for the corrected data at $t=1,1.5,2,2.5,3$ and $4 \mathrm{~s}$ (i.e., from $6 \%$ up to $25 \%$ of the freezing time of the droplet)

Fig. 5 Magnitude of the velocity along the symmetry line at $t=1,1.5,2,2.5,3$ and 4 s (i.e., from $6 \%$ up to $25 \%$ of the freezing time of the droplet)

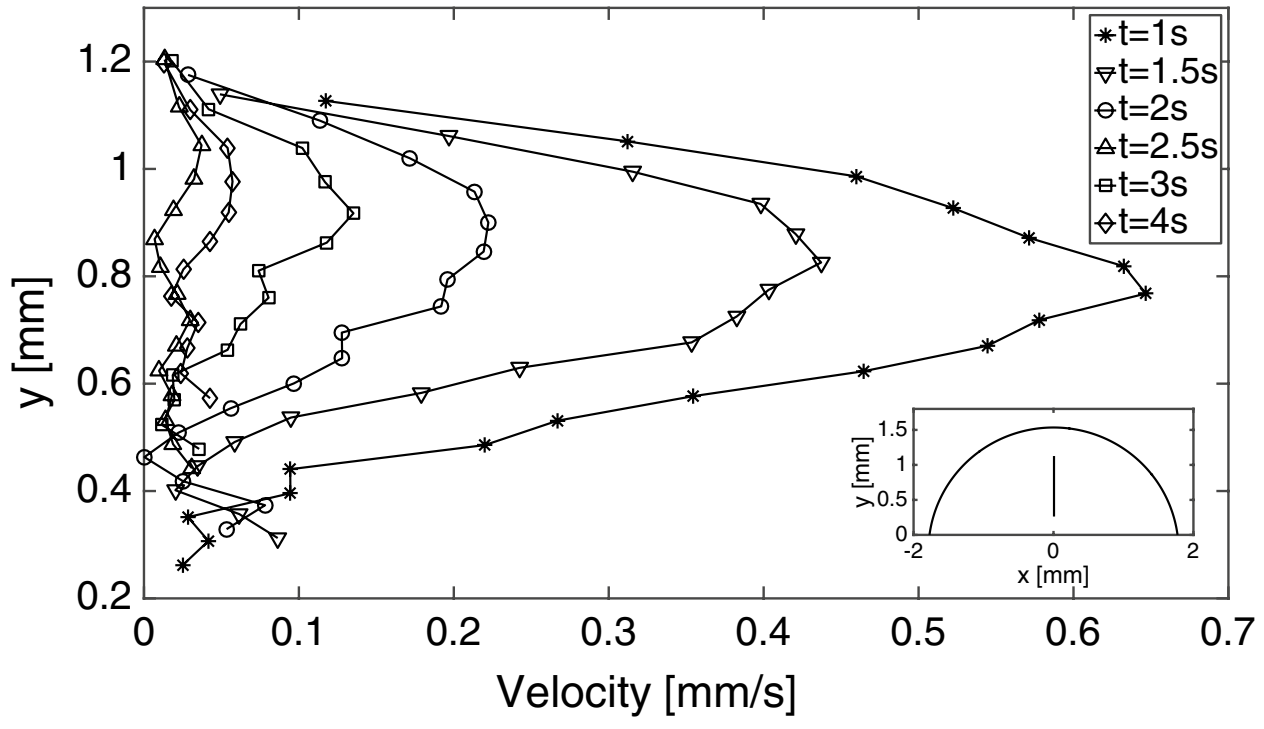

located higher up in the droplet will be pulled down along the interface towards the cooling surface and then move up again close to the center of the droplet. This behavior is observed up to $t=0.13 t_{\text {total }}$ in all cases, or up to the time $t=2 \mathrm{~s}$ presented in Fig. 4. Calculations of Marangoni number attained for these experiments suggest a number well 


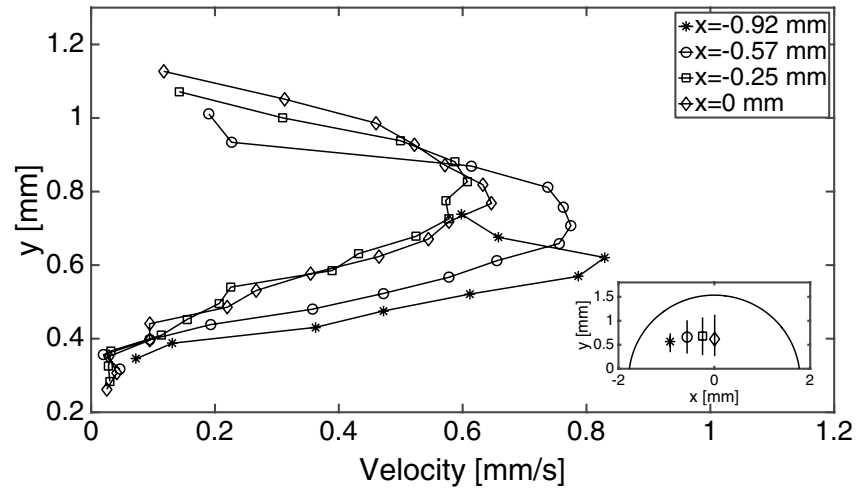

(a) $t=1 \mathrm{~s}\left(t=0.06 t_{\text {total }}\right)$

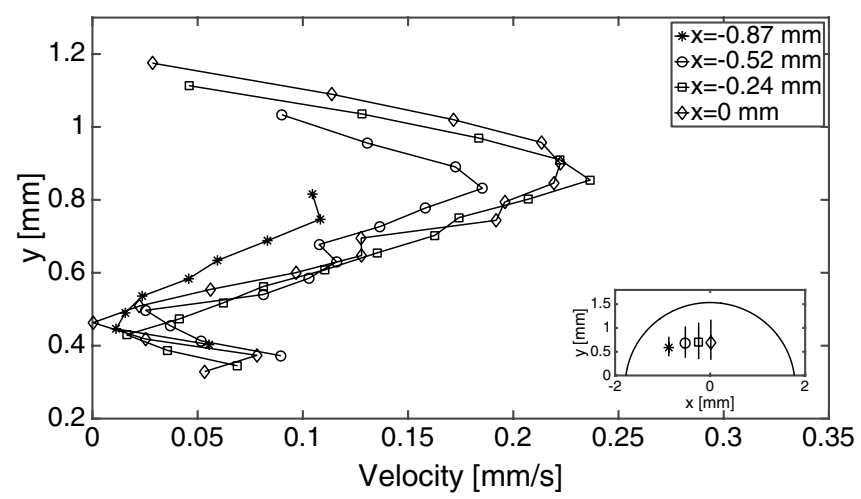

(c) $t=2 \mathrm{~s}\left(t=0.13 t_{\text {total }}\right)$

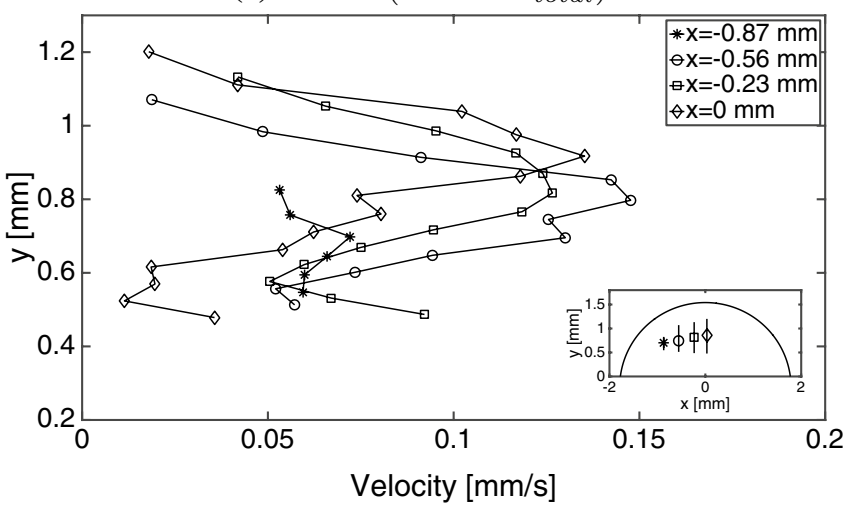

(e) $t=3 \mathrm{~s}\left(t=0.19 t_{\text {total }}\right)$

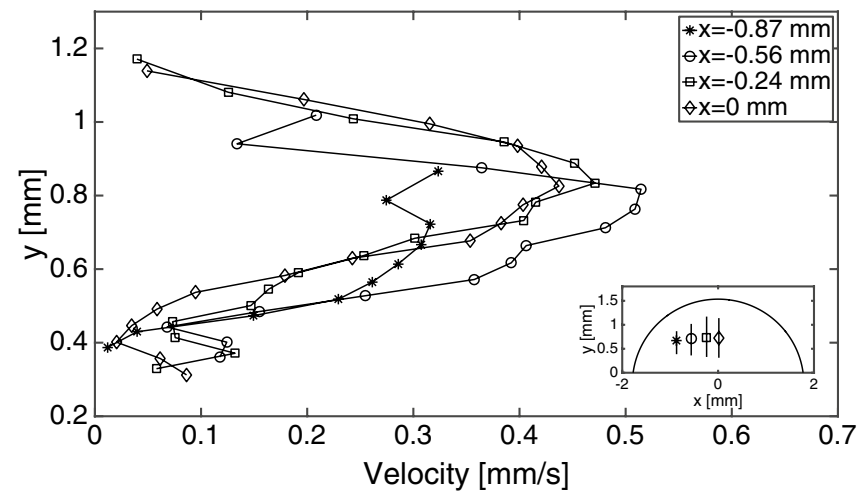

(b) $t=1.5 \mathrm{~s}\left(t=0.09 t_{\text {total }}\right)$

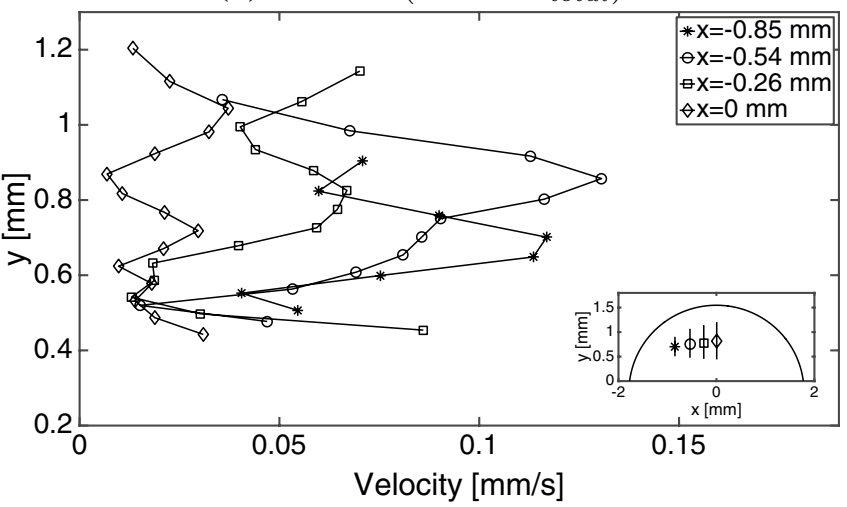

(d) $t=2.5 \mathrm{~s}\left(t=0.16 t_{\text {total }}\right)$

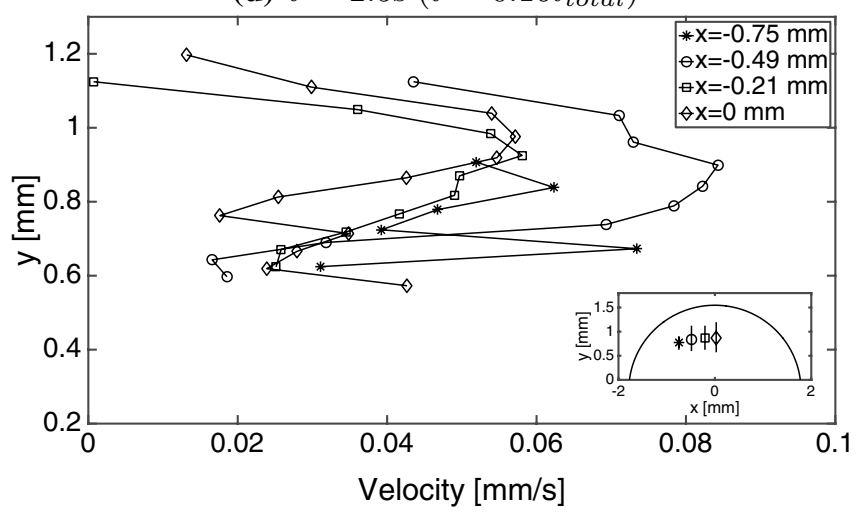

(f) $t=4 \mathrm{~s}\left(t=0.25 t_{\text {total }}\right)$

Fig. 6 Magnitude of the velocity along four lines at $t=1,1.5,2,2.5,3$ and 4 s (i.e., from $6 \%$ up to $25 \%$ of the freezing time of the droplet)

above 10,000 in the beginning of freezing, confirming large influence of Marangoni convection in ideal conditions (Pearson 1958). Marangoni convection in water droplets are, however, known to be highly sensitive for impurities and contaminations (Hu and Larson 2006; Xu and Luo 2007).

A possible reason for the interesting phenomenon that appears after $t=2.5 \mathrm{~s}$ in Fig. 4, as the flow moves in the direction from the cooling surface along the droplet surface to the top of the droplet (down along the symmetry line) might be due to increased effect of natural convection in the density inversion region of water together with decreased temperature difference at the surface. Even though the temperature distribution in the droplet is not investigated here, it can be assumed that the temperature of the water in the whole droplet at this time (at $t=4 \mathrm{~s}$ ) has dropped to a temperature close to zero. This assumption is based on the fact that since the mixing of water is great during these first seconds and that the cooling 
surface is much larger in comparison with the droplet, the water should have had time to cool off during this time period. Moreover, the flow in this time period show large similarities with the natural convection dominated flow modeled by Karlsson et al. (2018), both regarding direction of the vortices and the shape of the freezing front. Two differences between the simulations in Karlsson et al. (2018) and the presented experiments, is the direction of the flow in the first seconds of freezing, as well as the fact that the highest magnitude is retrieved in the absolute beginning of freezing, and not a few seconds into the freezing process as observed in the simulations. This reasoning suggest that natural convection is not the only mechanism inducing internal flow in the freezing droplet. After this point the flow is much slower due to the equalized temperatures in and around the droplet and also the viscosity in the water increases (an increase of about $80 \%$, from $T=21^{\circ} \mathrm{C}$ to $T=0{ }^{\circ} \mathrm{C}$ ) hence this will make the movement of the particles to decrease. As already mentioned, the analysis of the data ends at $t=0.25 t_{\text {total }}$ due to the relatively large formation of ice, which at this point spreads the laser sheet significantly. To conclude this section, note that the internal flow behavior in case 1 has been observed in all of the 10 cases studied in this paper when the time is scaled with the full freezing time.

\subsection{Comments on the frost formation}

Even though the frost formation is not, in detail, investigated here, some comments about its impact on the experiments should be made. How much frost that is actually created during the experiments is not measured. The time and relative humidity is monitored so that similar conditions are created at each experiment. If too much frost is added to the surface, the light from the laser will be spread out resulting in poor quality of the images retrieved as the droplet freezes. If too little frost is added to the surface, the droplet will not freeze and get super cooled instead. Also, based on visual observations more frost on the surface results in droplets with larger contact angles and longer freezing times. Since the surface is very smooth the frost initiates the ice nucleation that starts the freezing of the droplets. The conclusion is, therefore, that the frost is required to start the nucleation in the droplets, but based on the droplets seen in this work, it is at the expense of repeatability in appearances of the droplets (i.e., droplet height and droplet radius) and because of this also the freezing time of the droplet. More research of the impact of the frost on the freezing of droplets has to be made, but since the interest in this paper is of the inner flow, the current knowledge of the frost is considered sufficient.

\section{Conclusions}

In this work the internal flow within freezing water droplets was investigated using particle image velocimetry. It has been shown that the method proposed can be used to disclose the velocity field in freezing water droplets. To overcome the problems with the refraction at the droplet surface a correction method was used that maps the velocity vectors from the original particle images directly onto the object plane without image restoration. The time frame of interest is the first $25 \%$ of the freezing process, since this is when the characteristic vortices appear and the velocity of the water is at maximum due to the large temperature differences in and around the droplet. After this time period the temperature differences are small resulting in reduced velocity of the water and no vortices and also, the ice formation in the droplet spreads the light too much after this point resulting in images not suitable for analysis. Therefore, the results presented in this work only covers this time frame. When using the correction method the inner flow close to the centre of the droplet is well restored and the vortices are easy to track, but the flow close to the droplet surface is not retrieved. Initially the water is moving in the direction from the top of the droplet along the interface towards the cooling surface and then it is moving up again close to the center of the droplet, symmetrical on both sides of the centerline. This indicates that the flow is caused by the Marangoni effect due to the large temperature differences in and around the droplet. After a few seconds the flow has shifted in the opposite direction due to equalized temperatures at the surface and due to an increased effect of natural convection in the density inversion region of water. To the authors best knowledge, this is the first time that this change in flow direction has been visualized in freezing water droplets. To freeze the water droplets a frost layer was created using humidified air. The time and relative humidity was monitored so that similar conditions were created in each experiment. This was sufficient for this work, but the creation of frost is interesting and should be investigated further.

Acknowledgements Open access funding provided by Luleå University of Technology.

Open Access This article is distributed under the terms of the Creative Commons Attribution 4.0 International License (http://creativeco mmons.org/licenses/by/4.0/), which permits unrestricted use, distribution, and reproduction in any medium, provided you give appropriate credit to the original author(s) and the source, provide a link to the Creative Commons license, and indicate if changes were made. 


\section{References}

Anderson D, Grae Worster M, Davis S (1996) The case for a dynamic contact angle in containerless solidification. J Cryst Growth 163:329-338

Bhardwaj R, Fang X, Attinger D (2009) Pattern formation during the evaporation of a colloidal nanoliter drop: a numerical and experimental study. New J Phys 11:075020

Chaudhary G, Li R (2014) Freezing of water droplets on solid surfaces: an experimental and numerical study. Exp Therm Fluid Sci 57:86-93

Christy J, Sefiane K, Munro E (2010) A study of the velocity field during evaporation of sessile water and water/ethanol drops. J Bionic Eng 7:321-328

Coleman H, Steele W (2009) Experimentation, validation, and uncertainty analysis for engineers. Wiley, Hoboken

Enríquez OR, Marín AG, Winkels KG, Snoeijer JH (2012) Freezing singularities in water drops. Phys Fluids 24:091102

Hao P, Lv C, Zhang X (2014) Freezing of sessile water droplets on surfaces with various roughness and wettability. Appl Phys Lett 104:161609

Hu H, Larson R (2006) Marangoni effect reverses coffee-ring depositions. J Phys Chem B 110:7090-7094

Huang L, Liu Z, Liu Y, Gou Y, Wang L (2012) Effect of contact angle on water droplet freezing on a cold flat surface. Exp Therm Fluid Sci 40:74-80

Incropera F, Dewitt D, Bergman T, Lavine A (2013) Principles of heat and mass transfer. Wiley, New York

Jin Z (2008) Experimental investigations on micro-scale thermal fluid phenomena by using advance flow diagnostic techniques. $\mathrm{PhD}$ thesis, Iowa State University

Jin Z, Dong Q, Jin S, Yang Z (2012) Visualization of the freezing and melting process of a small water droplet on a cold surface. In: Proceedings of 2012 International conference on fluid dynamics and thermodynamics technologies (FDTT 2012), Singapore, vol 33

Jin Z, Jin S, Yang Z (2013a) An experimental investigation into the icing and melting process of a water droplet impinging onto a superhydrophobic surface. Sci China Phys Mech Astron 56(11):2047-2053

Jin Z, Jin S, Yang Z (2013b) Visualization of icing process of a water droplet impinging onto a frozen cold plate under free and forced convection. J Vis 16:13-17

Jin Z, Sui D, Yang Z (2015) The impact, freezing, and melting processes of a water droplet on an inclined cold surface. Int J Heat Mass Transf 90:439-453

Kang K, Lee S, Lee C, Kang I (2004) Quantitative visualization of flow inside an evaporating droplet using the ray tracing method. Meas Sci Technol 15:1104-1112

Karlsson L, Ljung AL, Lundström T (2018) Modelling the dynamics of the flow within freezing water droplets. Heat Mass Transf 54(12):3761-3769

Kawanami T, Yamada M, Fukusako S (1997) Solidification characteristics of a droplet on a horizontal cooled wall. Heat Transf Jpn Res 26(7):469-483
Liu Z, Zhang X, Wang H, Meng S, Cheng S (2007) Influences of surface hydrophilicity on frost formation on a vertical cold plate under natural convection conditions. Exp Therm Fluid Sci 31:789-794

Ljung AL, Lindmark E, Lundström T (2015) Influence of plate size on the evaporation rate of a heated droplet. Dry Technol 33(15-16): 1963-1970

Ma L, Liu Y, Hu H (2019) An experimental investigation on wind driven droplet moving on surfaces with different wettabilities. In: AIAA SciTech Forum 7-11 January 2019, San Diego, California

Marín AG, Enríquez OR, Brunet P, Colinet P, Snoeijer JH (2014) Universality of tip singularity formation in freezing water drops. Phys Rev Lett 113(5):054301

Minor G, Oshkai P, Djilali N (2007) Optical distortion correction for liquid droplet visualization using the ray tracing method: further considerations. Meas Sci Technol 18(11):L23-L28

Nauenberg M (2014) Theory and experiments on the ice-water front propagation in droplets freezing on a subzero surface. Eur J Phys 37(4):045102

Oberli L, Caruso D, Hall C, Fabretto M, Murphy PJ, Evans D (2014) Condensation and freezing of droplets on superhydrophobic surfaces. Adv Colloid Interface Sci 210:47-57

Pearson J (1958) On convection cells induced by surface tension. J Fluid Mech 4(5):489-500

Ruiz O, Black W (2002) Evaporation of water droplets placed on a heated horizontal surface. J Heat Transf 124:854-863

Schetnikov A, Matiunin V, Chernov V (2015) Conical shape of frozen water droplets. Am J Phys 83(36):36-38

Snoeijer J, Brunet P (2012) Pointy ice-drops: how water freezes into a singular shape. Am J Phys 80:764-771

Thielicke W, Stamhuis E (2014) PIVlab-towards user friendly, affordable and accurate digital particle image velocimetry in MATLAB. J Open Res Softw 2:30

Westerweel J (2000) Theoretical analysis of the measurement precision in particle image velocimetry. Exp Fluids 29(Suppl 1):S003-S012

Xu X, Luo J (2007) Marangoni flow in an evaporating water droplet. Appl Phys Lett 91:124102

Zhang X, Wu X, Min J (2017) Freezing and melting of a sessile water droplet on a horizontal plate. Exp Therm Fluid Sci 88:1-7

Zhang X, Liu X, Wu X, Min J (2018a) Experimental investigation and statistical analysis of icing nucleation characteristics of sessile water droplets. Exp Therm Fluid Sci 99:26-34

Zhang X, Liu X, Wu X, Min J (2018b) Simulation and experiments on supercooled sessile water droplet freezing with special attention to supercooling and volume expansion effects. Int J Heat Mass Transf 127(Part A):975-985

Publisher's Note Springer Nature remains neutral with regard to jurisdictional claims in published maps and institutional affiliations. 